\title{
Bundle of Joy: Improving Prenatal Well-being with Gratitude and Mindfulness
}

\author{
Karen O' Leary \\ School of Applied Psychology, UCC
}

\section{Feeling the weight}

Your feet, hands and belly are swollen. Your back is sore and there is almost constant pressure on your bladder. At times you feel absolutely exhausted. That's right, you're pregnant. Your body is doing amazing things; your baby is growing and wriggling about. You are filled with a new sense of expectancy and love, but you have also realised something. Pregnancy is not quite the 9 months of joy and wonder you thought it would be. You can deal with the physical complaints because you believe that they happen to everyone. The stress and sadness you are feeling is quite another thing. At times you feel like crying or giving up. There are many possible reasons for this. Maybe it is because your life has changed more than you are comfortable with. You may receive little or no help and support from your family, or your partner shows little interest in the pregnancy. Work may feel more stressful. You are worried about the future, the birth, your finances, or your life. Maybe for any or for all of these reasons, life has lost some of its colour and joy. You think to yourself, "that's not meant to happen when I'm pregnant, is it?"

For many women, low levels of well-being, characterised by stress, anxiety and unhappiness, are common and pervasive in pregnancy. Well-being involves being healthy and happy; it involves mental and physical aspects. Low levels of well-being are problematic for the expectant mother because they increase her risk of depression and engaging in an unhealthy behaviour, such as smoking. There are also subsequent effects for the developing foetus. For example, low maternal well-being during pregnancy can result in pregnancy complications, low birth weight, and premature birth. These birth outcomes can subsequently impact on infant and child development; so low prenatal well-being goes beyond simply feeling 'a bit down', and can have serious consequences. In Ireland, our maternity services provide high levels of prenatal care to approximately 70,000 expectant mothers each year. These services mainly monitor and foster the mother and baby's physical health and well-being but there is very little focus on fostering and promoting mental health during pregnancy. So, what can you do? How can you maintain or increase your well-being while you're pregnant? 


\section{Improving Well-being}

As a pregnant woman you can engage in activities to improve your well-being. You can do pregnancy yoga, pregnancy pilates or go for an "expectant mother" spa package. Yes, these activities can boost your mood, but are these activities really that accessible to most women? Firstly they all cost money. A 6 week pregnancy yoga class in Cork city costs $€ 70$, while a pregnancy spa package can set you back $€ 155$. What if you cannot afford this or you can only afford it once in the 9 months of pregnancy? What if you can afford it but just don't have the time to go? As a working, pregnant mother who already has a toddler running around, being able to go to the local yoga class is not exactly feasible.

This is where my research in health and positive psychology comes in. Health psychology has a long established tradition of examining changes and differences in health and wellbeing. In this case, my health focus is in the context of pregnancy. Positive psychology is the study of factors that allow us to thrive and live meaningful lives. It is not a happyology, as sometimes suggested, with a lot of pop psychologists running around telling everyone to 'smile more, it will make you feel better'. It is a scientific approach, which adopts a positive view of human functioning. The idea that we can foster and improve people's lives, rather than only supporting people in difficulty, is core in positive psychology. This counters the traditional view of pregnancy as a period of vulnerability where women may experience stress or depression and need treatment. A number of positive psychological interventions or exercises have been developed to improve well-being. The two types of interventions I use in my research are based on the positive constructs of gratitude and mindfulness. These constructs have a long history in people's private practice and in research.

\section{Feeling Grateful, Feeling Mindful}

Gratitude involves an appreciation of the positive in life. It can involve anything from a delicious home cooked meal to receiving a favour from a friend. The most widely studied gratitude-based intervention is the gratitude diary; this is sometimes referred to as 'counting your blessings'. In this exercise people are asked to think back over their day and write down things they feel grateful for. The main requirement is that they truly feel grateful for what they write down.

Mindfulness involves being fully aware of your thoughts, feelings and sensations in the present moment, in a non-judgmental way. In a sense, all internal and external stimuli are acknowledged but are not judged as positive or negative, they are simply accepted. This is not always an easy thing to do and mindfulness is a skill that must be practiced. Mindfulness has its roots in the Buddhist tradition. It shares a focus with positive psychology on cultivating positive aspects within oneself to bring about greater well-being. 
Studies examining both gratitude and mindfulness interventions have consistently demonstrated benefits for well-being. Both types of intervention can reduce stress, anxiety, depression and physical symptoms. They can also increase happiness, life satisfaction and physical health. Strangely, despite how much we know about the benefits of gratitude and mindfulness for physical and mental health, their usefulness has yet to be fully examined during pregnancy. As we noted earlier, improvements in well-being would benefit both the mother and child during pregnancy. So we need to find short, easy and cost-effective interventions that are readily accessible to all pregnant women. We then need to prove that they work! My research is focused on these two challenges.

\section{My Research}

My research involves two main studies to develop and test the usefulness of gratitude and mindfulness exercises during pregnancy. In the first study I developed two new interventions, one based on mindfulness and one based on gratitude. The gratitude exercise involves writing and thinking about things that you are grateful for. The mindfulness exercise focuses on writing and thinking about your thoughts, feelings and sensations in the present moment. An important part of this mindfulness exercise is that you are thinking but not dwelling or judging anything you think or feel. Both of these exercises can be completed at home in 15 minutes. In the first study non-pregnant women used the interventions 3 to 4 times a week, for 1 month. The women were randomly assigned to one of the intervention groups or a control group. The control group did not do the gratitude or mindfulness exercises during the study. The aim was to see which intervention was most effective before using it with the pregnant group. From this study, I found that combining elements of both exercises has the greatest potential to improve well-being.

The second study examines the effect of a new intervention on prenatal well-being. This exercise combines aspects of both gratitude and mindfulness. The impact of the exercise on well-being is examined in comparison to standard prenatal care. Although not yet completed, provisional results suggest that this 15-minute exercise can reduce levels of stress, depression and improve happiness and life satisfaction among pregnant women. This indicates that the exercise has the potential to provide a quick, easy and cost-effective way to maintain and enhance well-being during pregnancy. As there are currently no programs or interventions in place to promote mental well-being during pregnancy this could be especially beneficial for expectant mothers and their developing babies.

I would like to thank my supervisor Dr Samantha Dockray, School of Applied Psychology, for her support and advice, and also The O'Connor Scholarship for funding received. I would also like to thank Dr Mairead O' Riordan, Professor Louise Kenny and Professor John Higgins, Department of Obstetrics and Gynaecology, for support provided in conducting this research. 